\title{
Esteroides en extubación: ¿A quién? ¿Cómo? ¿Cuándo?
}

Steroids post-extubation: Who? How? When?

Esteróides na extubação: quem? Como e quando?

Job Heriberto Rodríguez Guillén, ${ }^{*}$ José Manuel Lomelí Terán, ${ }^{\star}$ Enrique Monares Zepeda, ${ }^{\ddagger}$ Manuel Poblano Morales, ${ }^{*}$ Héctor Fernando Hernández Ruiz ${ }^{\S}$

\section{RESUMEN}

El fracaso en la extubación es una complicación de alto riesgo para los pacientes en las Unidades de Cuidados Intensivos. El edema laríngeo y el estridor laríngeo han sido estudiados como causas que propician el fracaso en la extubación. Parte de las estrategias para la prevención del fracaso en la extubación y el manejo del estridor o edema laríngeo es el uso de esteroides. La discrepancia entre el tiempo, la dosis, el paciente candidato y el tipo de esteroide obligan a crear una propuesta que estandarice su utilización. La presente revisión se enfoca en identificar a aquellos pacientes que se benefician del uso de esteroides, el tipo de esteroide que se debe utilizar, el momento y la dosis correcta. Palabras clave: Esteroides, estridor laríngeo, edema laríngeo, extubación.

\section{ABSTRACT}

Post-extubation failure is a high risk complication for patients in intensive care unit. Laryngeal edema and laryngeal stridor have been studied as causes of post-extubation failure. Part of the strategies for the prevention of postextubation failure and management of stridor or laryngeal edema is the use of steroids. The discrepancy between the time, the dos, the patient and the type of steroid forces to create a strategy that standardizes its use. This review focuses on identify patients who benefit from the use of steroids, type of steroid that should be uses, timing and correct dose.

Keywords: Steroids, laryngeal stridor, laryngeal edema, extubation.

\section{RESUMO}

O fracasso na extubação é uma complicação de alto risco para pacientes em unidades de terapias intensivas. O edema e o estridor laríngeo foram estudados como causas que propician a falha na extubação. Parte das estratégias para prevenção da falha na extubação e manejo do estridor ou edema laríngeo é o uso de esteróides. A discrepância entre o tempo, a dose, o paciente candidato e o tipo de esteróide nos obriga a criar uma proposta padrão de utilização. A presente revisão se concentra em identificar os pacientes que são beneficiados com o uso de esteróides, o tipo de esteróide que deve ser usado, o momento e a dose correta.

Palavras-chave: Esteróides, estridor laríngeo, edema laríngeo, extubação.

\section{INTRODUCCIÓN}

El proceso de extubación es un paso crítico para el manejo de los pacientes en las Unidades de Cuidados Intensivos; este proceso puede convertirse en una complicación grave por la obstrucción de la vía aérea. Una causa frecuente de dicha obstrucción en los pacientes recientemente extubados es el edema laríngeo, que se manifiesta clínicamente por estridor y dificultad respiratoria. Bajo la hipótesis de que el edema causante del estridor es secundario a la inflamación e isquemia producidas por la presión del tubo endotraqueal a la laringe, los esfuerzos para prevenirlo se han enfocado en el empleo de corticosteroides, fundamentado principalmente en sus propiedades antiinflamatorias. El estridor se presenta hasta en $37 \%$ de los pacientes que llegan a la Unidad de Cuidados Intensivos (UCl),

\footnotetext{
* Hospital H+ Querétaro.

₹ Hospital San Ángel Inn Universidad.

$\S$ Facultad de Medicina UNAM.
}

Recepción: 22/07/2019. Aceptación: 15/08/2019.

Este artículo puede ser consultado en versión completa en www.medigraphic.com/medicinacritica siendo poco previsible la necesidad de reintubación. De acuerdo con la literatura, esta posibilidad oscila en un porcentaje muy variable, que va de 10 a $100 \%$, lo cual conlleva a un incremento en la estancia hospitalaria y en la morbimortalidad. Por lo anterior, está claro que se requiere de una intervención profiláctica ante el riesgo tan poco predecible y el impacto tan alto que puede desencadenar. ${ }^{1}$

Por todo esto, es pertinente revisar la mejor evidencia disponible que sustente a quién, cómo y cuándo usar esteroides para prevenir esta condición.

\section{Análisis de la literatura}

Existen pocos ensayos clínicos acerca de la relación entre corticosteroides y edema laríngeo después de la extubación. En este trabajo, revisamos la metodología de dos de los más grandes y de mejor calidad que existen hasta ahora: el trabajo publicado por François y su equipo, ${ }^{2}$ y el de Darmon y su grupo de investigadores. $^{3}$

Ambos ensayos son aleatorizados, paralelos, multicéntricos, doble ciego y controlados mediante placebo. Asimismo, son similares en su tamaño de muestra, los desenlaces evaluados y los objetivos (Tabla 1). No obstante, la diferencia más importante entre ellos es que, por un lado, el publicado por Darmon evalúa los efectos de dexametasona una hora antes de la extubación, y por el otro, el de François valora la metilprednisolona 12 horas antes de la extubación en dosis que divide cada cuatro horas.

Cada uno de los autores proporciona algunas definiciones. Para Darmon, el edema menor lo refiere como la presencia de estridor y de signos de obstrucción de la vía aérea superior. El primero lo definió como aquél confirmado por laringoscopia, el cual requería reintubación (sin especificar explícitamente si debían existir algunas manifestaciones clínicas). Las definiciones de François son más completas; este autor determinó como edema laríngeo a la presencia de obstrucción de la vía aérea superior; al edema menor lo caracterizó como estridor asociado con distrés respiratorio, el cual necesitó de atención médica; al edema mayor como un distrés respiratorio severo que necesitó reintubación secundaria a la obstrucción de la vía aérea superior visualizada con laringoscopia, y una fase inspiratoria prolongada con utilización de los músculos accesorios respiratorios fue la definición del distrés respiratorio. 
A diferencia del trabajo de François, Darmon no menciona el tratamiento de quienes desarrollaron edema, donde la terapéutica fue dejada al criterio del médico tratante.

Salvo por una mayor cantidad de mujeres en el grupo placebo que en el de dexametasona, los participantes del trabajo de Darmon fueron similares (Tabla 2). En el trabajo de François, más pacientes del grupo metilprednisolona fueron intubados vía nasotraqueal que los del grupo placebo (Tabla 3).

\section{RESULTADOS}

Darmon encontró que más pacientes del grupo placebo desarrollaron edema, con $4.8 \%$ en comparación con el grupo de dexametasona con $3.1 \%$; esto no representó significancia estadística. Las reintubaciones fueron menos frecuentes en el grupo de dexametasona que en el grupo placebo, con $2 / 11(18.1 \%)$ y $5 / 17$ (29.4\%), respectivamente (Tabla 4). François reportó que $3 \%$ de los pacientes que recibieron metilprednisolona desarrollaron edema frente a $22 \%$ del grupo placebo $(p<0.001)$. El edema mayor y la reintubación representaron $9 \%$ en el grupo metilprednisolona, y $18 \%$ en el grupo placebo $(p=0.68)$ (Tabla 5).

El estudio de Darmon reportó que el principal factor de riesgo para presentar edema laríngeo fue la intubación mayor a 36 horas $(7.2 \%$ en comparación con $0.9 \%$ de las intubaciones menores a 36 horas [ $<0.001]$ ); además ningún paciente con intubación menor a 36 horas requirió reintubación. El género femenino fue el segundo factor de riesgo $(70 \%)$, manifestando que quienes tuvieron edema laríngeo eran principalmente mujeres $(p<0.05)$. En el estudio de François, el género femenino representó el mayor riesgo (HR 2.02, IC 95\% 1.31 a 3.11); el traumatismo como motivo de ingreso a la UCI (OR 2.12, IC 95\% 1.29 a 3.49), la duración de intubación < 7 días (OR 1.77, IC 95\% 1.15 a 2.73) y la razón entre altura/diámetro de tubo (OR 0.97, IC 95\% 0.96 a 0.99 ) fueron los factores relacionados.

Tabla 1: Características de los estudios evaluados en esta revisión.

\begin{tabular}{|c|c|}
\hline Característica & Darmon, 1992 \\
\hline Objetivo & $\begin{array}{l}\text { Determinar los posibles factores de riesgo para la aparición de ede- } \\
\text { ma laríngeo después de la extubación y evaluar la potencial eficacia } \\
\text { de los corticosteroides para reducir su incidencia }\end{array}$ \\
\hline Desenlaces & $\begin{array}{l}\text { Edema laríngeo menor o mayor que ocurriera dentro de las } 24 \text { horas } \\
\text { postextubación* }\end{array}$ \\
\hline Diseño & $\begin{array}{l}\text { ECA, paralelo, multicéntrico, doble ciego (pacientes, médicos tratan- } \\
\text { tes, investigadores) y controlado con placebo }\end{array}$ \\
\hline Grupos & $\begin{array}{l}\text { Grupo de intervención: dexametasona. Grupo control: placebo } \\
\text { idéntico }\end{array}$ \\
\hline \multicolumn{2}{|l|}{ Intervención } \\
\hline Fármaco & Dexametasona fosfato sódico \\
\hline Vía & IV \\
\hline Posología & $8 \mathrm{mg}$ una hora antes de la extubación \\
\hline Otros procedimientos & $\begin{array}{l}\text { Durante la estancia hospitalaria, el neumotaponador del tubo fue } \\
\text { revisado cada } 6 \text { horas con la técnica de menor fuga. Todos los } \\
\text { pacientes fueron divididos según la duración de su intubación para e } \\
\text { análisis: corta duración: < } 36 \text { horas; larga duración: > } 36 \text { horas }\end{array}$ \\
\hline Criterios de selección & $\begin{array}{l}\text { Criterios de elección: hombres y mujeres de } 15 \text { años o más hospita- } \\
\text { lizados en las UCls e intubados por vía orotraqueal o nasotraqueal. } \\
\text { Criterios de exclusión: menos de } 15 \text { años de edad, enfermedades o } \\
\text { cirugías otorrinolaringológicas durante la presentación, haber presen } \\
\text { tado edema laríngeo postextubación durante su estancia hospitalaria } \\
\text { actual o estar siendo tratados con corticosteroides o antiinflamatorios } \\
\text { o esteroideos. Criterios de eliminación del análisis: quienes no se } \\
\text { les extubó después de haber recibido dexametasona o placebo o si } \\
\text { habían requerido reintubación dentro de las } 24 \text { horas postextubación } \\
\text { por razones que no fueran edema laríngeo }\end{array}$ \\
\hline Población & 700 aleatorizados, 663 incluidos en el análisis \\
\hline
\end{tabular}

François, 2007

Poner a prueba la hipótesis de que el pretratamiento con corticosteroides 12 horas antes de la extubación puede prevenir eficientemente la aparición de edema laríngeo postextubación en adultos con ventilación mecánica de más de 36 horas en la UCl

Primario**: aparición de edema laríngeo menor o mayor dentro de 24 horas después de una extubación planeada. Otros desenlaces: eventos adversos

ECA, paralelo, multicéntrico, doble ciego (pacientes, enfermeras, médicos tratantes, investigadores) y controlado con placebo Grupo de intervención: metilprednisolona. Grupo control: placebo idéntico

Hemisuccinato de metilprednisolona

IV

$20 \mathrm{mg} 12$ horas antes de la extubación y cada 4 horas con la última administración inmediatamente antes de la extubación (dosis total: $80 \mathrm{mg}$ ) El tratamiento del edema laríngeo fue dejado a la discreción del médico tratante

El desenlace primario fue evaluado por un mismo investigador en cada unidad para minimizar la variabilidad diagnóstica

Criterios de elección: > 18 años, duración de ventilación mecánica $>36$ horas, extubación planeada durante su estancia hospitalaria. Criterios de exclusión: embarazadas, antecedentes de obstrucción postextubación de la vía aérea superior, enfermedad o cirugía de garganta, traqueostomía, tratamiento crónico con antiinflamatorios no esteroideos o corticosteroides, incluido en otro ensayo o haber participado en éste. Criterios de eliminación del análisis: autoextubación o cancelación de extubación por deterioro

761 aleatorizados, 698 incluidos en el análisis

ECA = Ensayo clínico aleatorizado

IV = intravenosa.

*El edema laríngeo menor se definió como la presencia de estridor y/o signos de obstrucción aérea superior. El mayor se definió como un edema confirmado por laringoscopia directa que requería reintubación.

** El edema laríngeo se definió como obstrucción de la vía aérea superior. El edema menor corresponde a estridor asociado con distrés respiratorio que necesitó atención médica. El edema mayor fue un distrés respiratorio severo que necesitó reintubación secundaria a obstrucción de la vía aérea superior visualizada con laringoscopia. El distrés respiratorio fue caracterizado por una fase inspiratoria prolongada con utilización de músculos accesorios de la respiración. 
Tabla 2: Resultados de Darmon, 1992.

\begin{tabular}{|c|c|c|}
\hline \multirow[b]{2}{*}{ Características } & \multicolumn{2}{|c|}{ Darmon, 1992} \\
\hline & $\begin{array}{l}\text { Dexametasona } \\
\quad(n=348)\end{array}$ & $\begin{array}{l}\text { Placebo } \\
(n=352)\end{array}$ \\
\hline \multicolumn{3}{|l|}{ Edad: media $\pm \mathrm{DE}$} \\
\hline Intubación $<36$ horas & $49 \pm 18.8$ & $47.7 \pm 19.4$ \\
\hline Intubación > 36 horas & $55.4 \pm 17.9$ & $59.4 \pm 19.8$ \\
\hline Mujeres: n (\%) & $138(39.6)$ & $157(44.6)$ \\
\hline \multicolumn{3}{|c|}{$\begin{array}{l}\text { SAPS al momento de intubación: } \\
\text { mediana (rango) }\end{array}$} \\
\hline Intubación $<36$ horas & $8(1,24)$ & $9(2,23)$ \\
\hline Intubación > 36 horas & $12(2,27)$ & $12(2,27)$ \\
\hline \multicolumn{3}{|c|}{ Dificultades para intubar: $\mathrm{n}(\%)$} \\
\hline No & $312(89.6)$ & $322(91.4)$ \\
\hline Sí & $21(6)$ & $20(5.6)$ \\
\hline Desconocido & $15(4.3)$ & $10(2.8)$ \\
\hline \multicolumn{3}{|c|}{$\begin{array}{l}\text { Duración de intubación: días } \\
\text { promedio } \pm D E\end{array}$} \\
\hline Intubación $<36$ horas & No reportado & No reportado \\
\hline Intubación > 36 horas & $9.6 \pm 9.7$ & $10.3 \pm 10.9$ \\
\hline \multicolumn{3}{|l|}{ Diagnóstico de ingreso: $n$} \\
\hline Sobredosis & 86 & 106 \\
\hline Postoperado & 109 & 95 \\
\hline Insuficiencia respiratoria & 45 & 55 \\
\hline Shock & 36 & 44 \\
\hline Enfermedad neurológica & 43 & 35 \\
\hline Otros & 29 & 17 \\
\hline \multicolumn{3}{|l|}{ Vía de intubación: n (\%) } \\
\hline Oral & $131(37.6)$ & $125(35.5)$ \\
\hline Nasal & 217 (62.3) & $227(64.4)$ \\
\hline \multicolumn{3}{|l|}{$\begin{array}{l}\text { Número de intubaciones } \\
\text { previas: } n\end{array}$} \\
\hline 1 & 306 & 318 \\
\hline 2 & 31 & 26 \\
\hline$>2$ & 11 & 8 \\
\hline
\end{tabular}

SAPS = Simplified Acute Physiology Score; DE = desviación estándar.

\section{¿Qué dice el resto de la evidencia?}

En 2017, fue publicada una revisión sistemática en CHEST por Kurinyama y colaboradores. ${ }^{4}$ En él se incluyeron 11 ensayos: en cinco se utilizaron dexametasona; en cuatro, metilprednisolona, y en dos, hidrocortisona. Dos fueron considerados con un riesgo general de sesgo bajo: los de François (2007) y Lee (2007). ${ }^{5}$ En la revisión, el uso de corticosteroides redujo el riesgo de eventos postextubación (obstrucción de la vía aérea superior, edema laríngeo o estridor) en $60 \%$ y el riesgo de reintubación en $58 \%$ (Tabla 6).

En el metaanálisis, se dividieron a los estudios en dos: un grupo en el que los ensayos seleccionaron a sujetos de alto riesgo, determinado mediante la prueba de fuga del manguito taponador (cuff o neumotaponador) y otro grupo de ensayos con pacientes no seleccionados. La razón de riesgos (RR) para los eventos postextubación en el grupo de pacientes de alto riesgo fue de 0.34 (IC 95\%, 0.24 a 0.48 ), mientras que en el grupo de no seleccionados, esta asociación no fue significativa (RR 0.62, IC 95\%
Tabla 3: Resultados de François, 2007.

\begin{tabular}{|c|c|c|}
\hline \multirow[b]{2}{*}{ Características } & \multicolumn{2}{|c|}{ François, 2007} \\
\hline & $\begin{array}{l}\text { Metilprednisolona } \\
\quad(n=380)\end{array}$ & $\begin{array}{l}\text { Placebo } \\
(\mathrm{n}=381)\end{array}$ \\
\hline \multicolumn{3}{|l|}{$\begin{array}{l}\text { Edad: años, mediana (rango } \\
\text { intercuartílico) }\end{array}$} \\
\hline Mujeres: $\mathrm{n}(\%)$ & $142(37)$ & $135(35)$ \\
\hline $\begin{array}{l}\text { SAPS II al momento de intubación: } \\
\text { mediana (rango intercuartílico) }\end{array}$ & $40(30 \text { a } 51)^{*}$ & $38(29 \text { a } 48)^{*}$ \\
\hline \multicolumn{3}{|l|}{ Dificultades para intubar: $\mathrm{n}(\%)$} \\
\hline No & $375(99)$ & $370(97)$ \\
\hline Sí & $5(1)$ & $11(3)$ \\
\hline \multicolumn{3}{|l|}{ Duración de intubación: $\mathrm{n}(\%)$} \\
\hline$<7$ días & $194(51)$ & $187(49)$ \\
\hline$\geq 7$ días & $186(49)$ & $194(51)$ \\
\hline \multicolumn{3}{|l|}{ Motivo de ingreso: $\mathrm{n}(\%)$} \\
\hline Médico & $240(63)$ & $229(60)$ \\
\hline Quirúrgico & $74(19)$ & $74(19)$ \\
\hline Traumatismo & $66(17)$ & $78(20)$ \\
\hline \multicolumn{3}{|l|}{ Vía de intubación: n (\%) } \\
\hline Oral & $343(90)$ & $359(94)$ \\
\hline Nasal & $37(10)$ & $21(6)$ \\
\hline \multicolumn{3}{|l|}{ Número de intubaciones previas: $n(\%)$} \\
\hline 1 & $318(84)$ & $317(83)$ \\
\hline 2 & $55(14)$ & $48(13)$ \\
\hline$>2$ & $7(2)$ & $16(4)$ \\
\hline
\end{tabular}

SAPS = Simplified Acute Physiology Score.

${ }^{*}$ Datos faltantes de un paciente.

Tabla 4: Edema laríngeo y reintubación con dexametasona.

\begin{tabular}{lcc}
\hline & \multicolumn{2}{c}{ Darmon, 1992} \\
\cline { 2 - 3 } Características & Dexametasona $(\mathrm{n}=348)$ & Placebo $(\mathrm{n}=352)$ \\
\hline Edema laríngeo: & & \\
$\mathrm{n}(\%[$ [IC $95 \%])$ & $1(0.7[0.02$ a 3.6]) & $2(1.2[0.15$ a 4.4$])$ \\
$\quad$ Intubación $<36$ horas & $10(5.7[2.8$ a 10.5$])$ & $15(8.7[5$ a 14.1$])$ \\
Intubación $>36$ horas & $11(3.1)$ & $17(4.8)$ \\
$\quad$ Total & $2 / 11(18.1)$ & $5 / 17(29.4)$ \\
Reintubaciones: $\mathrm{n} / \mathrm{N}(\%)$ &
\end{tabular}

IC $95 \%$ = intervalo de confianza 95\%.

Tabla 5: Edema laríngeo y reintubación con metilprednisolona.

\begin{tabular}{lccc}
\hline & \multicolumn{2}{c}{ François, 2007} & \\
\cline { 2 - 3 } Desenlace & $\begin{array}{c}\text { Metilprednisolona } \\
(\mathrm{n}=355)\end{array}$ & $\begin{array}{c}\text { Placebo } \\
(\mathrm{n}=343)\end{array}$ & $\mathrm{p}$ \\
\hline Primario & & & \\
Edema laríngeo: $\mathrm{n}(\%)$ & $344(97)$ & $267(78)$ & \\
$\quad$ No & $11(3)$ & $76(22)$ & \\
$\quad$ Sí & & & 0.0001 \\
Severidad & $10(91)$ & $62(82)$ & \\
$\quad$ Menor & $1(9)$ & $14(18)$ & \\
$\quad$ Mayor & & & \\
Reintubaciones: $\mathrm{n}(\%)$ & $342(96)$ & $317(92)$ & \\
$\quad$ No & $13(4)$ & $26(8)$ & \\
$\quad$ Sí & & & \\
Contexto de reintubación & $11(85)$ & $9(35)$ & \\
$\quad$ Sin edema laríngeo & $1(8)$ & $14(54)$ & \\
$\quad$ Reintubación asociada a & & & \\
edema laríngeo & &
\end{tabular}




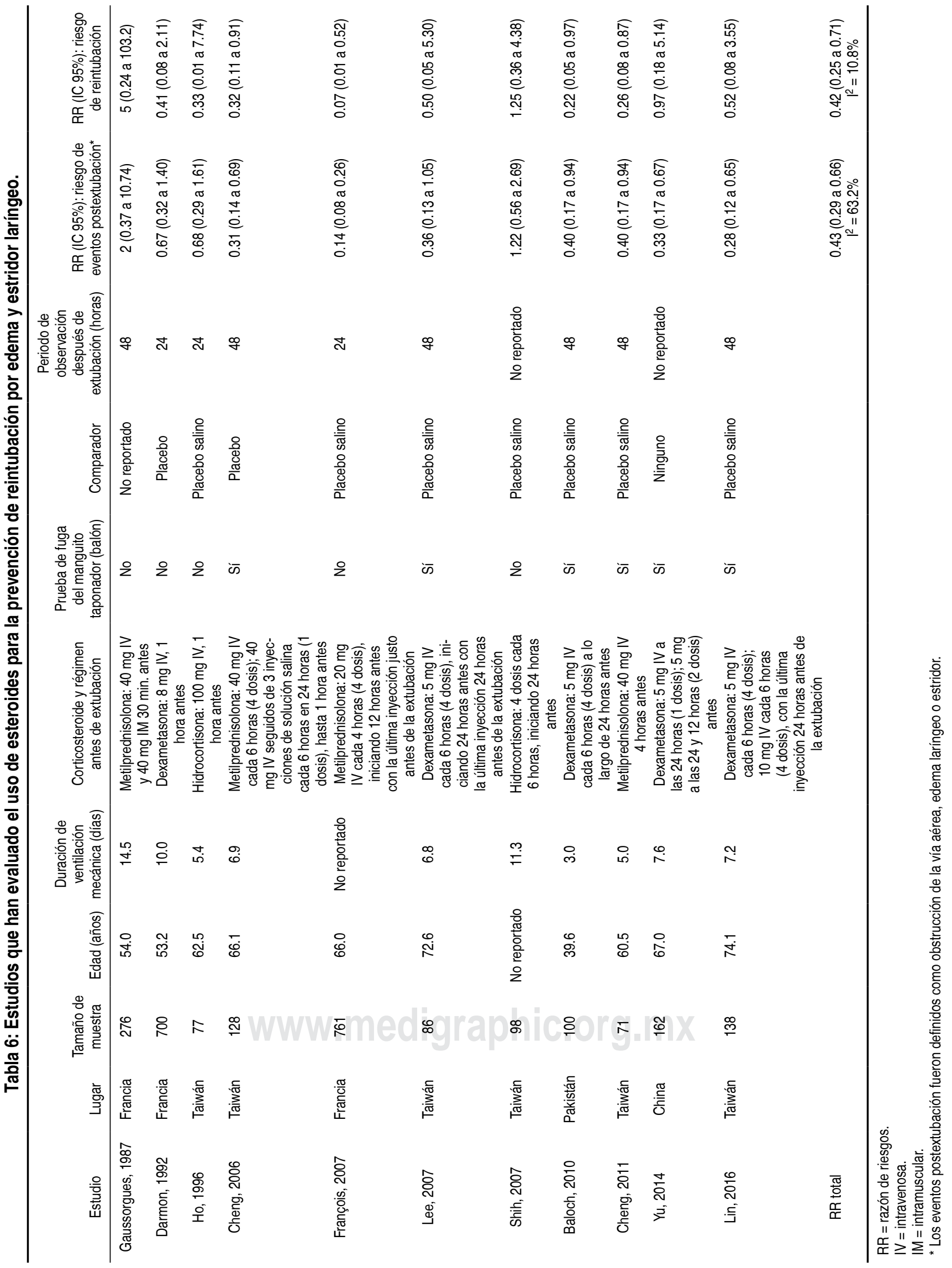


Test de fuga

1. Aspire las secreciones traqueales y orales

2. Con el neumotaponador inflado, registre el Vt inspiratorio

3. Corrobore similitud con el Vt-espiratorio

4. Desinfle el neumotaponador

5. Registre el Vt-espiratorio durante los próximos 6 ciclos y promedie los 3 valores más bajos

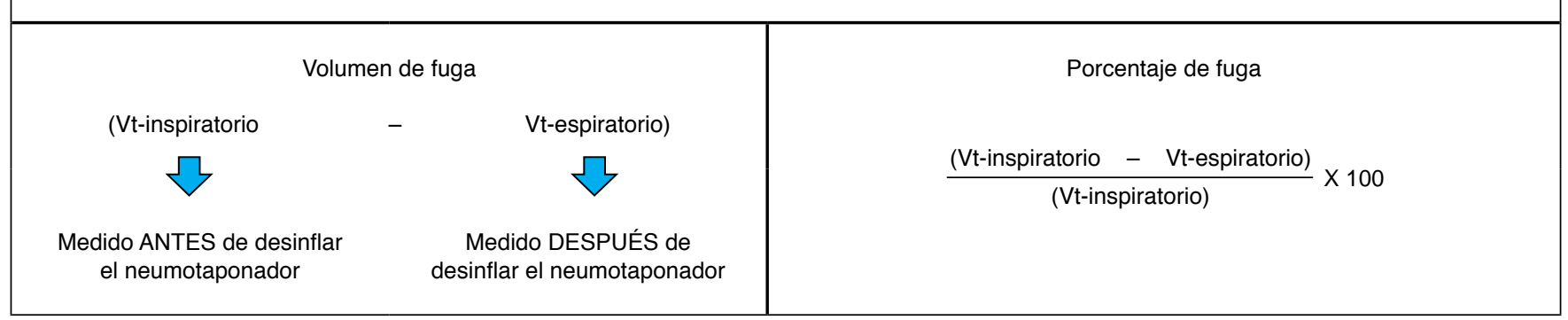

Figura 1: Test de fuga. Vt = volumen tidal.

Protocolo de extubación y prevención de reintubación

Paciente despierto $+\mathrm{SpO}_{2} \geq 92 \%+\mathrm{FiO}_{2} \leq 40 \%+\mathrm{PEEP} \leq 8+\mathrm{Sin}$ vasopresores

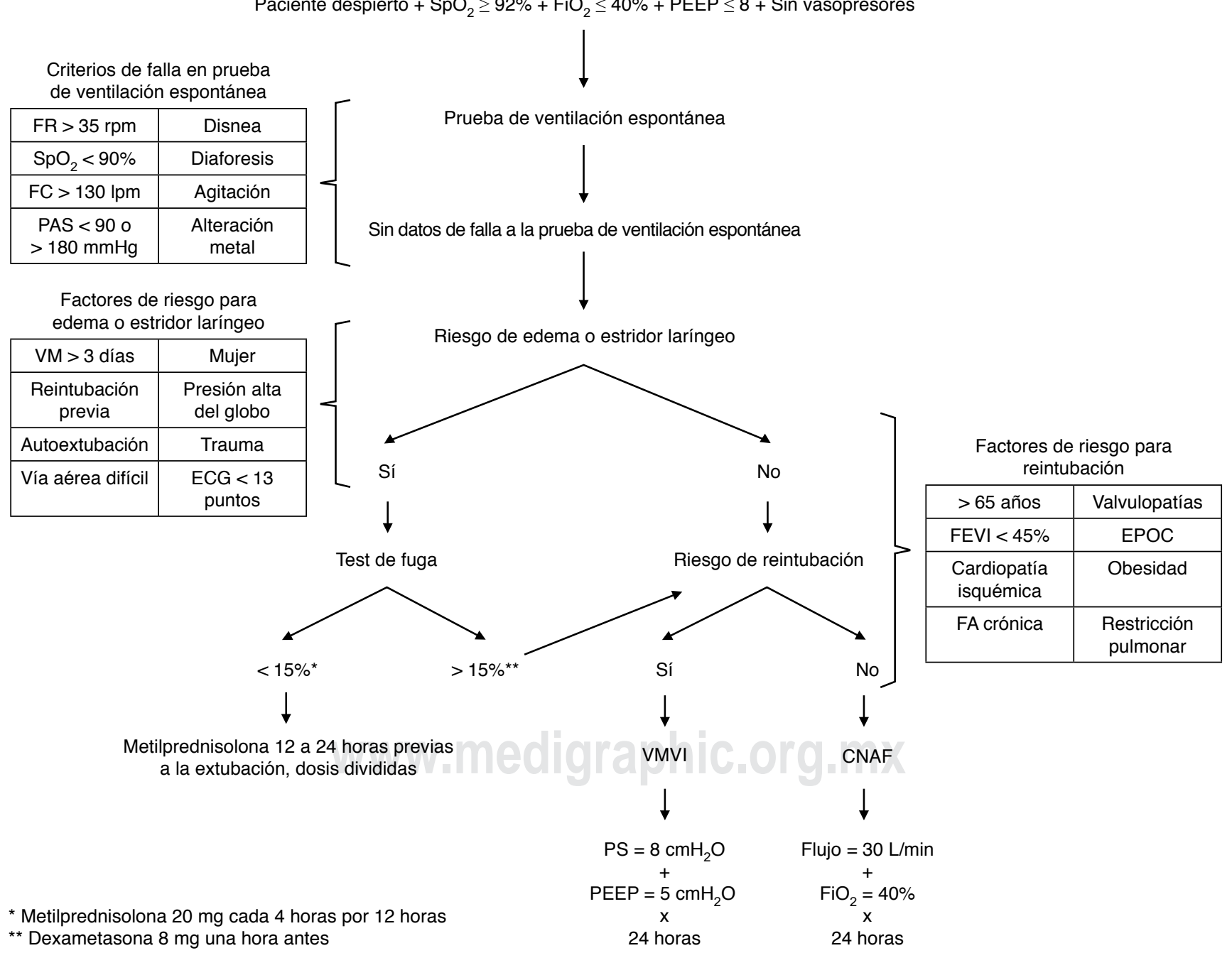

Figura 2: Protocolo de extubación y prevención de reintubación.

$\mathrm{SpO}_{2}=$ saturación de oxígeno; $F \mathrm{Fi}_{2}=$ fracción inspirada de oxígeno; $P E E P=$ presión positiva al final de la espiración; $F R=$ frecuencia respiratoria; $F C=$ frecuencia cardiaca; $P A S$ = presión arterial sistólica; $V M=$ ventilación mecánica; $E C G$ = escala de coma de Glasgow; $F E V I$ = fracción de expulsión del ventrículo izquierdo; EPOC = enfermedad pulmonar obstructiva crónica; $P S$ = presión soporte; $V M N I=$ ventilación mecánica no invasiva; $C N A F$ = cánulas nasales de alto flujo; $F A=$ fibrilación auricular. 
0.24 a 1.61). Respecto a la reintubación, la $R R$ del grupo de alto riesgo fue de 0.35 (IC 95\% 0.20 a 0.64 ) y 0.53 (IC $95 \% 0.15$ a 1.89 ) en el grupo de no seleccionados.

Los eventos adversos fueron infrecuentes. En ningún ensayo ocurrió hemorragia o hiperglucemia y sólo un paciente presentó una infección.

\section{DISCUSIÓN}

Considerando la evidencia, quedan pocas dudas sobre la efectividad de los corticosteroides. Sin embargo, aún hay preguntas por responder: ¿cuál corticosteroide ha de utilizarse?, ¿en qué momento y cuántas veces?, ¿dosis? y ¿a qué paciente se le ha de administrar?

Es importante conocer los factores de riesgo basales (género femenino, duración prolongada de la intubación, tubo endotraqueal grande, traumatismo como motivo de ingreso), pero ante todo hay que considerar que la variable que más predijo el riesgo de desarrollar edema, estridor o reintubación fue el volumen de fuga. Por lo tanto, es más importante la prueba de fuga que conocer el tipo de corticosteroide (Figura 1). ${ }^{6}$ Esta fuga se ha expresado como un valor absoluto o como un porcentaje del volumen tidal inspiratorio. ${ }^{7,8}$ Cuando el volumen de fuga es menor de 110-130 $\mathrm{mL}$ o menor de 10 a $15 \%{ }^{9,10}$ del volumen tidal, el riesgo de estridor postextubación es significativamente elevado.

En cuanto al número de dosis, una revisión de 2011 encontró que las dosis múltiples 12 a 24 horas antes de la extubación tienen mejores resultados que las dosis únicas. ${ }^{11}$ La recomendación actual de la ATS/ACCP (American Thoracic Society/American College of Chest Physicians) es administrar el fármaco al menos cuatro horas antes de extubar. ${ }^{12}$ Sin embargo, esta guía fue publicada antes de la revisión sistemática de CHEST, la cual no encontró que el tiempo desde la primera dosis hasta la extubación modificara la aparición del edema.

El tiempo de administración debe considerar que los mejores resultados se han encontrado cuando se inicia 12 a 24 horas antes. Autores como Hyzy y su grupo sugieren el empleo de metilprednisolona $20 \mathrm{mg}$ cada cuatro horas para un total de cuatro dosis antes de la extubación, esquema que cumple con las recomendaciones antes mencionadas. ${ }^{13}$ Sin embargo, se requieren más estudios que evalúen el régimen óptimo. No obstante, el uso de hidrocortisona no debe priorizarse, puesto que es el corticosteroide menos estudiado y los trabajos que lo han evaluado son de mala calidad. Al elegir entre metilprednisolona o dexametasona, se debe recordar que ambas parecen dar resultados similares.

\section{CONCLUSIÓN}

Actualmente no se cuenta con una prueba estandarizada para la identificación de pacientes de alto riesgo de desarrollar edema y estridor laríngeo. Por lo tanto, la decisión de iniciar corticosteroides debe realizarse de forma individualizada, dando un mayor peso al resultado de la prueba de fuga del neumotaponador y los factores de riesgo relacionados con el edema laríngeo y estridor (Figuras 1 y 2). En presencia de insuficiencia respiratoria, la reintubación debe realizarse sin demora.

\section{AGRADECIMIENTOS}

Al capítulo de Ventilación Mecánica del Colegio Mexicano de Medicina Crítica.

A Jule Santos, Vicepresidenta ABRAMEDE-DF, por su apoyo en la traducción al portugués.

\section{BIBLIOGRAFÍA}

1. Jaber S, Jung B, Chanques G, Bonnet F, Marret E. Effects of steroids on reintubation and post-extubation stridor in adults: meta-analysis of randomised controlled trials. Crit Care. 2009;13(2):R49.

2. Francois B, Bellissant E, Gissot V, Desachy A, Normand S, Boulain $\mathrm{T}$, et al. 12-h pretreatment with methylprednisolone versus placebo for prevention of post-extubation laryngeal edema: a randomized double-blind trial. Lancet. 2007;369:1083-1089.

3. Darmon JY, Rauss A, Dreyfuss D, Bleichner G, Elkharrat D, Schlemmer B, et al. Evaluation of risk factors for laryngeal edema after tracheal extubation in adults and its prevention by dexamethasone. A placebo-controlled, double-blind, multicenter study. Anesthesiology. 1992;369:245-251.

4. Kuriyama A, Umakoshi, Sun R. Prophylactic corticosteroids for prevention of postextubation stridor and reintubation in adults: a systematic review and meta-analysis. Chest. 2017;151:1002-1010.

5. Lee CH, Peng MJ, Wu CL. Dexamethasone to prevent postextubation airway obstruction in adults: a prospective, randomized, double-blind, placebo-controlled study. Crit Care. 2007;11:R72.

6. Argalious M. The cuff leak test: does it "leak" any information? Repir Care. 2012;57:2136-2137.

7. Miller R, Cole R. Association between reduced cuff leak volume and postextubation stridor. Chest. 1996;110:1035-1040.

8. Jaber S, Chanques G, Matecki S, Ramonatxo M, Vergne C, Souche B, et al. Postextubation stridor in intensive care unit patients. Risk factors evaluation and importance of the cuff-leak test. Intensive Care Med. 2003;29:69-74.

9. DeBast Y, DeBacker D, Moraine J, Lemaire M, Vandenborght $\mathrm{C}$, Vincent JL. The cuff leak test to predict failure of tracheal extubation for laryngeal edema. Intensive Care Med. 2002;28:1267-1272.

10. Sandhu R, Pasquale M, Miller K, Wasser T. Measurement of endotracheal tube cuff leak to predict postextubation stridor and need for reintubation. J Am Coll Surg. 2000;190:682-687.

11. Khemani R, Randolph a, Markovitz B. Corticosteroids for the prevention and treatment of post-extubation stridor in neonates, children and adults. Cochrane Database Syst Rev. 2009;3:CD001000.

12. Girard T, Alhazzani W, Kress J, Ouellette D, Schmidt G, Truwit $\mathrm{J}$, et al. An official American Thoracic Society/American College of Chest Phyisicians Clinical Practice Guideline: liberation from mechanical ventilation in critically ill adults. Rehabilitation protocols, ventilator liberation protocols, and cuff leak test. $A m \mathrm{~J}$ Respir Crit Care Med. 2017;195:120-133.

13. Hyzy R. Extubation management in the adult intensive care unit. Post TW, ed. UpToDate. Waltham, MA: UpToDate Inc. [Accessed on July 22, 2019] Available in: https://uptodate.com

Correspondencia:

Job Heriberto Rodríguez Guillén

E-mail: jobrodriguez@hmasqueretaro.mx 